\title{
Predictors of postoperative hypocalcemia occurring after a total thyroidectomy: results of prospective multicenter study
}

Vitalijus Eismontas ${ }^{1 *}$, Algirdas Slepavicius ${ }^{1}$, Vinsas Janusonis ${ }^{1}$, Paulius Zeromskas ${ }^{1}$, Virgilijus Beisa², Kestutis Strupas ${ }^{2}$, Zilvinas Dambrauskas ${ }^{3}$, Antanas Gulbinas ${ }^{3}$ and Arvydas Martinkenas ${ }^{4}$

\begin{abstract}
Background: Thyroid surgeries are among the most common operations performed in the world. Hypocalcemia following total thyroidectomy is a common complication that is sometimes difficult to correct. The aim of this study is to determine the risk factors for hypocalcemia following total thyroidectomy and their clinical value.

Methods: From January 2015 through to April 2017, 400 patients were included in this prospective multicenter study. All patients underwent total thyroidectomy due to various thyroid diseases. The following risk factors were analyzed: pre-operative and post-operative biochemical blood parameters, clinical effects and factors related to surgery, the patient, and the disease.

Results: Post-operative hypocalcemia developed in 257 patients (64.2\%). Of them, 197 patients (76.7\%) were diagnosed with asymptomatic hypocalcemia. Clinical symptoms were present in 60 of the 257 patients with hypocalcemia (23.3\%). The statistically significant predictors of hypocalcemia were decreased calcium and ionized calcium pre-operatively $(p<0.001)$, parathyroid hormone on day one following surgery $(p<0.001)$, thyrotoxicosis $<10$ years before surgery (odds ratio $1.65,95 \% \mathrm{Cl} 1.01-2.70, p=0.046$ ), the number of parathyroid glands found during surgery (odds ratio $0.52,95 \% \mathrm{Cl} 0.38-0.70, p<0.001$ ), ligation of the trunk of the left inferior thyroid artery (odds ratio $2.04,95 \% \mathrm{Cl} 1.27-3.29, p=0.003$ ), ligation of the trunk of the right inferior thyroid artery (odds ratio 2.37, $95 \% \mathrm{Cl} 1.47-3.81, p<0.001$ ), and the number of transplanted parathyroid glands (odds ratio 1.87, 95\% Cl 1.12-2.97, $p=0.015$ ). In the multivariate analysis, age (odds ratio $1.05,95 \% \mathrm{Cl} 1.01-1.09, p=0.029$ ) and gender (odds ratio 5.94, $95 \% \mathrm{Cl} 1.13-31.26, p=0.035)$ were statistically significant predictors.

Conclusions: This study demonstrates that there is a number of different patient (gender, age, and duration of thyrotoxicosis $<10$ years before surgery) and surgical (number of parathyroid glands found during surgery, decreased calcium and ionized calcium before surgery, parathyroid hormone on day one following surgery, and ligation of the trunk of the left and right inferior thyroid artery) risk factors predictive of hypocalcemia following total thyroidectomy. Optimization of the surgical technique could possibly prevent the occurrence of hypocalcemia after total thyroidectomy in some cases; in other cases, identification of known risk factors post-operatively could permit early detection and effective treatment of these patients.
\end{abstract}

Keywords: Total thyroidectomy, Hypocalcemia, Thyroid surgery, Predictors

\footnotetext{
* Correspondence: eismontasv@yahoo.com

'Department of Abdominal and Endocrine Surgery, Klaipeda University

Hospital, Liepojos St. 41, 92288 Klaipeda, Lithuania

Full list of author information is available at the end of the article
}

(c) The Author(s). 2018 Open Access This article is distributed under the terms of the Creative Commons Attribution 4.0 International License (http://creativecommons.org/licenses/by/4.0/), which permits unrestricted use, distribution, and reproduction in any medium, provided you give appropriate credit to the original author(s) and the source, provide a link to the Creative Commons license, and indicate if changes were made. The Creative Commons Public Domain Dedication waiver (http://creativecommons.org/publicdomain/zero/1.0/) applies to the data made available in this article, unless otherwise stated. 


\section{Background}

Thyroid surgeries are among the most common in the world [1]. Thyroid surgery is the definitive management option for thyroid malignancies, and also for benign diseases such as multinodular goiter with compression symptoms [2]. Hypocalcemia following total thyroidectomy (TT) is a relatively frequent complication, which is sometimes difficult to correct. Temporary hypocalcemia occurs in $50-68 \%$ of post-TT patients [3, 4], while permanent hypocalcemia occurs in $3 \%$ of post-TT patients [5-7]. Temporary hypocalcemia is defined by various authors as a post-surgery decrease in calcium $(\mathrm{Ca})$, lasting for 6 to 12 months; permanent hypocalcemia is a post-TT decrease in Ca lasting for more than 12 months [8]. Post-TT hypocalcemia depends on a number of factors, including biochemical blood parameters before and after surgery, clinical effects and factors related to surgery, surgical technique, surgeon's experience, the patient, and the disease [9].

The aim of this study was to determine the risk factors for postoperative hypocalcemia following $\mathrm{TT}$ and their clinical value.

\section{Methods}

From January 2015 to April 2017, 400 patients underwent surgeries for various thyroid diseases at Klaipeda University Hospital, Lithuania and Vilnius University Hospital Santaros Klinikos, Lithuania. Permission from the Lithuanian Bioethics Committee was obtained for this prospective multicenter research (2014-12-02, No.: L-14-09/1, No.: L-14-09/2). All patients signed a consent form before surgery. The inclusion criteria for this study were thyroid nodules in 361 patients (90.3\%), thyroid carcinoma in 84 patients $(21 \%)$, thyroiditis in 37 patients (9.3\%). Some patients had more than one indication for surgery. The exclusion criteria for this study were previous hemithyroidectomy, lobectomy, resection of thyroid gland, and diagnosed pathology of parathyroid glands (PGs). In this study all operations were performed by a three high-volume thyroid surgeons. Various factors that may influence post-TT hypocalcemia were investigated, including pre-operative and post-operative biochemical blood parameters, clinical effects and factors related to surgery, the patient, and the disease. Postoperative hypocalcemia was diagnosed when the level of $\mathrm{Ca}$ in the blood was $<2.10 \mathrm{mmol} / \mathrm{L}$. Before surgery, $\mathrm{Ca}$, ionized calcium (iCa), parathyroid hormone (PTH), 25hydroxyvitamin D (25-hydroxy Vit D), and thyroid hormones were measured. Length of stay in hospital for patients following the surgery was 2 days. Measurements following TT on the first and second day at six o'clock in the morning were performed. On day one following the surgery, $\mathrm{Ca}$, $\mathrm{iCa}$, and $\mathrm{PTH}$ were measured. On day two following the surgery, $\mathrm{Ca}, \mathrm{iCa}$ were measured.
Clinical expression of post-operative hypocalcemia, and the time when the disease manifested, were assessed. The impact of surgery-related factors on post-operative hypocalcemia was analyzed, including the number of PGs observed during the surgery, number of autotransplanted PGs, and ligation of vessels supplying blood to the thyroid gland. Oral Ca with/without calcitriol were not routinely given unless patients had symptoms or serum $\mathrm{Ca}<2 \mathrm{mmol} / \mathrm{L}$. Oral Ca 1500-3000 mg with/ without calcitriol $0.25 \mathrm{mcg}$ twice daily were appointed for 2 weeks following the surgery. Calcitriol should be given in case a higher risk of postoperative hypocalcemia, including recurrent neck surgery, central neck dissection, intraoperative PG injury, autotransplanted PGs, also for patients with low postoperative PTH and 25-hydroxy Vit D deficiency.

All blood samples were taken from a peripheral vein. 25-hydroxy Vit D and PTH were measured with Cobas e411 analyzer (Roche Diagnostics, Mannheim, Germany). The normal range of 25-hydroxy Vit $\mathrm{D}$ in the blood is $75.00-106.75 \mathrm{nmol} / \mathrm{L}$ for men and 75.00-124.75 nmol/L for women. The normal PTH range is $15.00-65.00 \mathrm{pg} / \mathrm{mL}$. The level of $\mathrm{Ca}$ and $\mathrm{iCa}$ was determined with Architect ci8200 analyzer (Abbot, Wiesbaden, Germany).

The normal range of $\mathrm{Ca}$ in the blood is 2.10$2.55 \mathrm{mmol} / \mathrm{L}$ and the normal range of $\mathrm{iCa}$ in the blood is $1.00-1.30 \mathrm{mmol} / \mathrm{L}$.

\section{Statistical analysis}

Data analysis was performed using SPSS version 24.0 and MS Excel 2016. Univariate descriptive statistics were performed for the continuous data. Categorical data were summarized as frequencies and percentages. The chi-square test or the Fishers exact test was used to estimate the associations between categorical variables. Comparison of proportions between the groups was performed using the $\mathrm{z}$ test. After testing for normality, parametric or non-parametric tests were performed, as required. Comparison of health variables between hypocalcemia (no (0), yes (1)) groups was performed using the Students $t$ test and the Mann-Whitney test. ANOVA and the Kruskal-Wallis test was employed for comparison of two or more groups. Univariate binary logistic regression analysis was used to determine the relative significance of sociodemographic and perceived health variables as predictors of hypocalcemia. Variables associated with post-operative hypocalcemia in univariate analyses were incorporated into a multivariate logistic regression to identify independent risk factors for post-operative hypocalcemia following thyroidectomy. Multivariate logistic regression analysis, using the backward LR (Likelihood Ratio) test, was employed to remove variables with $p>0.1$. Odds ratios (ORs) of statistically significant predictors are presented with 95\% 
confidence intervals (CIs). A $p$ value of less than 0.05 was taken to indicate statistical significance.

\section{Results}

From January 2015 to April 2017, 400 patients underwent TT's. There were 349 women $(87.2 \%)$ and 51 men $(12.8 \%)$. The mean age was 57.2 years and the mean weight was $79.2 \mathrm{~kg}$. According to the data, post-operative hypocalcemia developed in 257 patients (64.2\%). Of them, 197 patients (76.7\%) were diagnosed with asymptomatic hypocalcemia. Clinical symptoms were observed in 60 patients $(23.3 \%)$ of the 257 patients with hypocalcemia. Numbness of fingers was observed in 58 patients $(22.6 \%$ of the patients with post-operative hypocalcemia). Chvostek's signs were present in 14 patients $(5.4 \%$ of the patients with post-operative hypocalcemia). Trousseau's signs were present in eight patients (3.1\% of the patients with post-operative hypocalcemia). In the group of patients with post-operative hypocalcemia, one symptom was present in 44 patients (17.1\%), two symptoms were present in 11 patients (4.3\%), and three symptoms were observed in five patients $(1.9 \%)$. See Tables 1 and 2 for details.

Comparison of the patients with diagnosed hypocalcemia following surgery and the patients without diagnosis of hypocalcemia showed that the following variables were significantly related to the development of hypocalcemia: decreased pre-operative $\mathrm{Ca}(p<0.001)$, and decreased pre-operative iCa $(p<0.001)$. Our study did not showed an increased risk of postoperative hypocalcemia in the case of malignancy: papillary carcinoma ( $p$ $=0.518)$, follicular carcinoma $(p=0.358)$, medullary carcinoma $(p=0.556)$, and bilateral central neck dissection $(p=1.000)$, wound hematoma $(p=0.753)$, bleeding during surgery $(p=1.000)$, revision for bleeding $(p=1.000)$. These data are presented in Table 2 .

Univariate analysis revealed that thyrotoxicosis $<10$ years before surgery was a predisposing factor for hypocalcemia (OR 1.65, 95\% CI 1.01-2.70, $p=0.046$ ). The number of PGs observed during surgery was also statistically associated with post-operative hypocalcemia (OR 0.52, 95\% CI 0.38-0.70, $p<0.001$ ). Ligation of the trunk of the left inferior thyroid artery (OR 2.04, 95\% CI 1.27-3.29, $p=0.003)$ and the trunk of the right inferior thyroid artery (OR 2.37, 95\% CI 1.47-3.82, $p<0.001$ ) were statistically significant factors related to post-operative hypocalcemia. The number of PGs autotransplanted during surgery was also significantly associated with post-TT hypocalcemia (OR 1.87, 95\% CI 1.12$2.97, p=0.015)$. Finally, PTH on day one following the surgery was a statistically significant predictor of post-operative hypocalcemia (OR 0.96, 95\% CI 0.94$0.97, p<0.001)$. This data is presented in Table 3 .
Table 1 Patient demographics and clinical characteristics

\begin{tabular}{|c|c|}
\hline Variable $^{a}$ & Value \\
\hline Gender, $n(\%)$ & $400(100)$ \\
\hline Female & $349(87.2)$ \\
\hline Male & $51(12.8)$ \\
\hline Age in years, mean (SD) & $57.2(13.4)$ \\
\hline Weight in kg, mean (SD) & $79.2(15.8)$ \\
\hline \multicolumn{2}{|l|}{ Thyrotoxicosis before surgery, $n(\%)$} \\
\hline No & $277(69.2)$ \\
\hline$<10$ years & $105(26.3)$ \\
\hline$\geq 10$ years & $18(4.5)$ \\
\hline \multicolumn{2}{|l|}{ Graves' disease, $n(\%)$} \\
\hline No & $379(94.7)$ \\
\hline Yes & $21(5.3)$ \\
\hline \multicolumn{2}{|l|}{ Recurrent goiter, $n(\%)$} \\
\hline No & $397(99.2)$ \\
\hline Yes & $3(0.8)$ \\
\hline \multicolumn{2}{|l|}{ Retrosternal goiter, n (\%) } \\
\hline No & $393(98.2)$ \\
\hline Yes & $7(1.8)$ \\
\hline \multicolumn{2}{|l|}{ Bone mass density, $\mathrm{T}$ level, $n$ (\%) } \\
\hline No & $394(98.5)$ \\
\hline Yes & $6(1.5)$ \\
\hline Preoperative calcium, mmol/L, mean (SD) & $2.36(0.10)$ \\
\hline MD (IQR) & $2.36(0.12)$ \\
\hline Preoperative ionized calcium, mmol/L, mean (SD) & $1.06(0.08)$ \\
\hline MD (IQR) & $1.04(0.07)$ \\
\hline Preoperative PTH, ng/L (pg/mL), mean (SD) & $57.71(22.27)$ \\
\hline $\mathrm{MD}(\mathrm{IQR})$ & $53.90(26.63)$ \\
\hline Preoperative 25 -hydroxyvitamin $\mathrm{D}, \mathrm{nmol} / \mathrm{L}$, mean (SD) & $45.27(22.03)$ \\
\hline $\mathrm{MD}(\mathrm{IQR})$ & $41.78(29.72)$ \\
\hline Preoperative TSH, mIU/L, mean (SD) & $0.94(1.22)$ \\
\hline $\mathrm{MD}(\mathrm{IQR})$ & $0.75(1.22)$ \\
\hline Preoperative FT3, pmol/L, mean (SD) & $4.87(2.36)$ \\
\hline MD (IQR) & $4.66(0.84)$ \\
\hline Preoperative FT4, pmol/L, mean (SD) & $13.31(2.69)$ \\
\hline MD (IQR) & $13.43(2.71)$ \\
\hline Thyroidectomy without lymphadenectomy, n (\%) & $400(100)$ \\
\hline No & $66(16.5)$ \\
\hline Yes & $334(83.5)$ \\
\hline
\end{tabular}

Thyroidectomy with one sided central lymphadenectomy, $n$ (\%)

No $344(86.0)$

Yes 56 (14.0)

Thyroidectomy with central lymphadenectomy on both sides, $n$ (\%)

No

$390(97.5)$

Yes

$10(2.5)$ 
Table 1 Patient demographics and clinical characteristics (Continued)

\begin{tabular}{l} 
Variable $^{\text {a }}$ \\
\hline Number of observed parathyroid glands, $n$ (\%) \\
1 \\
2 \\
3 \\
4 \\
Upper branch of left inferior thyroid artery, $n(\%)$ \\
Non-ligation \\
Ligation \\
Lower branch of left inferior thyroid artery, $n(\%)$ \\
Non-ligation \\
Ligation \\
Trunk of left inferior thyroid artery, $n$ (\%) \\
Non-ligation \\
Ligation
\end{tabular}

Upper branch of right inferior thyroid artery, $n$ (\%)

Non-ligation

Ligation

Lower branch of right inferior thyroid artery, $n$ (\%)

Non-ligation$$
\text { Ligation }
$$

Trunk of right inferior thyroid artery, $n$ (\%)

$$
\begin{array}{ll}
\text { Non-ligation } & 91(22.8) \\
\text { Ligation } & 309(77.2)
\end{array}
$$

Number of darkened parathyroid glands at the end of surgery, $n(\%)$

$$
0
$$$$
1
$$$$
2
$$$$
3
$$

Parathyroid gland hematoma, $n$ (\%)

$$
\begin{aligned}
& \text { No } \\
& \text { Yes }
\end{aligned}
$$

Number of autotransplanted parathyroid glands, $n$ (\%)$$
0
$$$$
1
$$$$
2
$$$$
3
$$

Adenomatous nodules, $n$ (\%)

$$
\begin{aligned}
& \text { No } \\
& \text { Yes } \\
& \text { Colloidal nodules, } n(\%)
\end{aligned}
$$

No

Yes
Value

$2(0.5)$

$8(2.0)$

$97(24.2)$

$208(52.0)$

$85(21.3)$

$363(90.7)$

$37(9.3)$

$366(91.5)$

$34(8.5)$

$90(22.5)$

$310(77.5)$

$358(89.5)$

$42(10.5)$

366 (91.5)

$34(8.5)$

305 (76.2)

$80(20.0)$

$9(2.3)$

$6(1.5)$

$379(94.7)$

$21(5.3)$

$332(83.0)$

54 (13.4)

$11(2.8)$

$3(0.8)$

334 (83.5)

$66(16.5)$

$130(32.5)$

$270(67.5)$
Table 1 Patient demographics and clinical characteristics (Continued)

\begin{tabular}{ll}
\hline Variable $^{a}$ & Value \\
\hline Papillary carcinoma, $n(\%)$ & $320(80.0)$ \\
No & $80(20.0)$ \\
Yes & \\
Follicular carcinoma, $n(\%)$ & $399(99.7)$ \\
No & $1(0.3)$ \\
Yes & \\
Medullary carcinoma, $n(\%)$ & $397(99.3)$ \\
No & $3(0.8)$ \\
Yes &
\end{tabular}

Anaplastic carcinoma, $n$ (\%)

No

$400(100)$

Autoimmune thyroiditis, $n$ (\%)

No

$363(90.7)$

Yes

$37(9.3)$

Calcium day one, mmol/L, mean (SD) $2.12(0.14)$

MD (IQR)

$2.11(0.20)$

$0.99(0.08)$

$0.99(0.10)$

$29.98(19.46)$

$29.06(27.61)$

$2.05(0.16)$

$2.06(0.22)$

$0.98(0.09)$

$0.98(0.11)$

MD (IQR)

396 (99.0)

Revision for bleeding, $n$ (\%)

No

$4(1.0)$

Wound infection, $n(\%)$

No

$400(100)$

Wound hematoma, $n(\%)$

$$
\text { No }
$$

389 (97.2)

Yes

$11(2.8)$

Bleeding during surgery, $n$ (\%)

No

399 (99.7)

Yes

$1(0.3)$

Clinical voice hoarseness, $n$ (\%)

No

$384(96.0)$

Yes

$16(4.0)$

Recurrent laryngeal nerve paralysis on one side, ORL, $n$ (\%)

$$
\text { No }
$$

$392(98.0)$

Yes

Recurrent laryngeal nerve paralysis on both sides, ORL, $n(\%)$

No

$400(100)$ 
Table 1 Patient demographics and clinical characteristics (Continued)

\begin{tabular}{ll}
\hline Variable $^{a}$ & Value \\
\hline Clinical manifestation of hypocalcemia, $n(\%)$ & $340(85.0)$ \\
None & $14(3.5)$ \\
Day one & $46(11.5)$ \\
Day two & \\
Hypocalcemia without clinical manifestation, $n$ (\%) & $203(50.7)$ \\
No & $197(49.3)$ \\
Yes & \\
Hypocalcemia - Chvostek's symptoms, $n(\%)$ & $386(96.5)$ \\
No & $14(3.5)$ \\
Yes & \\
Hypocalcemia - Trousseau's symptoms, $n$ (\%) & $392(98.0)$ \\
No & $8(2.0)$ \\
Yes & \\
Hypocalcemia - Numbness of fingers, $n(\%)$ & $342(85.5)$ \\
No & $58(14.5)$ \\
Yes & \\
Hypocalcemia, $n$ (\%) & $143(35.8)$ \\
No & $257(64.2)$ \\
Yes &
\end{tabular}

Treatment commenced in hospital with calcium and vitamin D preparations after surgery, $n(\%)$

$\begin{array}{ll}\text { None } & 161(40.3) \\ \text { Day } 1 & 15(3.8) \\ \text { Day } 2 & 33(8.3) \\ \text { Day } 3 & 5(1.3) \\ \text { On discharge } & 186(46.5)\end{array}$

a Continuous variables are presented as means and standard deviations (SD) \& medians $(M D)$ and interquartile ranges (IQR); categorical variables are presented as $n$ (\%). PTH Parathyroid hormone, TSH thyroid stimulating hormone; FT3 free triiodothyronine, FT4 free thyroxine,

ORL otorhinolaryngologist

Unlike the univariate analysis, multivariate analysis revealed that older age (OR 1.05, 95\% CI 1.01-1.09, $p=$ 0.029 ) and female gender (OR 5.94, 95\% CI 1.13-31.26, $p=0.035)$ were statistically significant independent predictors of post-operative hypocalcemia. See Table 4.

\section{Discussion}

Post-TT hypocalcemia is one of the most common complications [10]. According to the literature, post-TT hypocalcemia occurs in $50-68 \%$ of patients [3, 4]. In our study, post-TT hypocalcemia developed in $64.2 \%$ of the patients. In the literature, the majority of patients exhibit asymptomatic hypocalcemia and need no treatment, or only oral administration of $\mathrm{Ca}$ and vitamin $\mathrm{D}$ preparations [11]. In our study, 197 patients had asymptomatic hypocalcemia (49.3\% of all the patients who participated in the study, or $76.7 \%$ of the patients with post-operative hypocalcemia). Less than half of the patients received no treatment after TT $(161 ; 40.3 \%)$. The patients who received treatments were prescribed oral or intravenous preparations of $\mathrm{Ca}$ and vitamin $\mathrm{D}$, depending on the time of expression and intensity of clinical symptoms (Table 2). Overall, 60 patients (15\% of all the patients who participated in the study, or $23.3 \%$ of the patients with post-operative hypocalcemia) had one, two, or three clinical symptoms of hypocalcemia. In a multicenter study with 14,934 patients, Rosato et al. found that $10 \%$ of patients had symptomatic hypocalcemia [3]. However, that study included patients who had TT (64.3\%) and smaller-scale surgeries on the thyroid (35.7\%) [3]. In our study, decreased pre-operative $\mathrm{Ca}$ and $\mathrm{iCa}$ were statistically significant predictors of post-operative hypocalcemia $(p<0.001)$. Several studies have found that temporary post-operative hypocalcemia develops more often in patients who have a markedly decreased level of Ca before surgery [12-18]. However, a meta-analysis of six studies with 2493 patients did not reveal a statistically reliable association between pre-operative $\mathrm{Ca}$ and the frequency of temporary hypocalcemia [17-21]. A sharp decrease in $\mathrm{Ca}$ after TT is associated with temporary hypocalcemia [12, 22-24]. In a multicenter study with 1157 patients, Hallgrimsson et al. found that patients who experienced a $2-3 \%$ decrease in postoperative $\mathrm{Ca}$ in the $24 \mathrm{~h}$ following surgery, in comparison with the pre-operative level of $\mathrm{Ca}$, had a $94 \%$ chance of developing temporary hypocalcemia [25]. Changes in the levels of $\mathrm{Ca}$ in the blood during the first $24 \mathrm{~h}$ following surgery allow prediction of temporary hypocalcemia with $19-91 \%$ sensitivity [26-30]. A blood Ca concentration of $1.88 \mathrm{mmol} / \mathrm{L}$ or less during the first $24 \mathrm{~h}$ after surgery has been associated with permanent hypocalcemia [31]. Two other studies have demonstrated that there is an increased risk of developing permanent hypocalcemia if the level of $\mathrm{Ca}$ in the blood remains at $2 \mathrm{mmol} / \mathrm{L}$ or less, 1-3 weeks after surgery [32, 33]. Tartaglia et al. revealed that measurement of $\mathrm{iCa}$ was more reliable than measurement of $\mathrm{Ca}$ in post-TT patients, in the immediate and long-term follow-up [34].

Based on our data, decreased PTH during the first $24 \mathrm{~h}$ after surgery was a statistically reliable predictor of post-operative hypocalcemia $(p<0.001)$. In many other studies, a decrease in blood PTH following TT was found to result in possible development of temporary hypocalcemia [12, 35-39]. The systematic overview and metaanalysis conducted by Edafe et al. showed that patients with a decrease in post-operative $\mathrm{PTH}$, to a level of 6-35 pg/mL, $1 \mathrm{~h}$ to 1 day following surgery, had a 69$100 \%$ chance of developing temporary hypocalcemia [9].

According to our data, the number of PGs identified during the surgery was a statistically significant predictor 
Table 2 Patient characteristics

\begin{tabular}{|c|c|c|c|}
\hline \multirow[t]{2}{*}{ Variables $^{a}$} & \multicolumn{2}{|l|}{ Hypocalcemia } & \multirow[t]{2}{*}{$p$ value } \\
\hline & Yes & No & \\
\hline Gender, $n(\%)$ & 257 & 143 & \\
\hline Female & $221(63.3)$ & $128(36.7)$ & $x^{2}=1.02, \mathrm{df}=1, p=0.351$ \\
\hline Male & $36(70.6)$ & $15(29.4)$ & \\
\hline Age in years, mean (SD) & $57.8(13.2)$ & $56.0(13.8)$ & 0.207 \\
\hline Weight, kg., mean (SD) & $79.0(15.2)$ & $79.5(16.8)$ & 0.721 \\
\hline \multicolumn{4}{|l|}{ Thyrotoxicosis before surgery, $n(\%)$} \\
\hline No & $170(66.1)$ & $107(74.8)$ & $x^{2}=4.10, \mathrm{df}=2, p=0.129$ \\
\hline$<10$ years & $76(29.6)$ & $29(20.3)$ & \\
\hline$\geq 10$ years & $11(4.3)$ & $7(4.9)$ & \\
\hline \multicolumn{4}{|l|}{ Graves' disease, $n(\%)$} \\
\hline No & $241(93.8)$ & $138(96.5)$ & $x^{2}=1.38, \mathrm{df}=1, p=0.350$ \\
\hline Yes & $16(6.2)$ & $5(3.5)$ & \\
\hline \multicolumn{4}{|l|}{ Recurrent goiter, $n(\%)$} \\
\hline No & $254(98.8)$ & $143(100)$ & $x^{2}=1.68, \mathrm{df}=1, p=0.556$ \\
\hline Yes & $3(1.2)$ & 0 & \\
\hline \multicolumn{4}{|l|}{ Retrosternal goiter, $n$ (\%) } \\
\hline No & $250(97.3)$ & $143(100)$ & $x^{2}=3.96, \mathrm{df}=1, p=0.053$ \\
\hline Yes & $7(2.7)$ & 0 & \\
\hline \multicolumn{4}{|l|}{ Bone mass density, T level, $n$ (\%) } \\
\hline No & $394(98.5)$ & $394(98.5)$ & $x^{2}=0.54, \mathrm{df}=1, p=0.671$ \\
\hline Yes & $6(1.5)$ & $6(1.5)$ & \\
\hline Preoperative calcium, mmol/L, mean (SD) ${ }^{b}$ & $2.35(0.09)$ & $2.39(0.11)$ & $<0.001$ \\
\hline $\mathrm{MD}(\mathrm{IQR})$ & $2.35(0.11)$ & $2.39(0.12)$ & \\
\hline Preoperative ionized calcium, mmol/L, mean $(S D)^{b}$ & $1.04(0.07)$ & $1.08(0.10)$ & $<0.001$ \\
\hline $\mathrm{MD}(\mathrm{QQR})$ & $1.03(0.07)$ & $1.07(0.09)$ & \\
\hline Preoperative PTH, ng/L (pg/mL), mean $(S D)^{b}$ & $57.86(22.18)$ & $57.43(22.50)$ & 0.855 \\
\hline $\mathrm{MD}(\mathrm{IQR})$ & $54.17(26.38)$ & $53.65(26.05)$ & \\
\hline Preoperative 25 -hydroxyvitamin $\mathrm{D}, \mathrm{nmol} / \mathrm{L}$, mean $(\mathrm{SD})^{\mathrm{c}}$ & $44.22(22.97)$ & $47.16(20.17)$ & 0.053 \\
\hline $\mathrm{MD}(\mathrm{IQR})$ & $40.35(30.47)$ & $46.40(26.69)$ & \\
\hline Preoperative $\mathrm{TSH}, \mathrm{mIU} / \mathrm{L}$, mean $(\mathrm{SD})^{\mathrm{c}}$ & $1.12(1.33)$ & $0.96(0.97)$ & 0.355 \\
\hline $\mathrm{MD}(\mathrm{IQR})$ & $0.80(1.20)$ & $0.70(1.17)$ & \\
\hline Preoperative $\mathrm{FT} 3$, pmol/L, mean $(\mathrm{SD})^{\mathrm{b}}$ & $4.85(2.73)$ & $4.90(1.50)$ & 0.854 \\
\hline $\mathrm{MD}(\mathrm{IQR})$ & $4.60(0.83)$ & $4.71(0.93)$ & \\
\hline Preoperative FT4, pmol/L, mean (SD) ${ }^{b}$ & $13.53(2.47)$ & $13.67(3.05)$ & 0.626 \\
\hline $\mathrm{MD}(\mathrm{IQR})$ & $13.45(2.92)$ & $13.40(2.36)$ & \\
\hline \multicolumn{4}{|l|}{ Thyroidectomy without lymphadenectomy, n (\%) } \\
\hline No & $48(18.7)$ & $18(12.6)$ & $x^{2}=2.47, \mathrm{df}=1, p=0.124$ \\
\hline Yes & $209(81.3)$ & $125(87.4)$ & \\
\hline \multicolumn{4}{|c|}{ Thyroidectomy with one sided central lymphadenectomy, $n$ (\%) } \\
\hline No & $216(84.0)$ & $128(89.5)$ & $x^{2}=2.28, \mathrm{df}=1, p=0.175$ \\
\hline Yes & $41(16.0)$ & $15(10.5)$ & \\
\hline \multicolumn{4}{|c|}{ Thyroidectomy with central lymphadenectomy on both sides, $n$ (\%) } \\
\hline No & $250(97.3)$ & $140(97.9)$ & $x^{2}=0.15, \mathrm{df}=1, p=1.000$ \\
\hline
\end{tabular}


Table 2 Patient characteristics (Continued)

\begin{tabular}{|c|c|c|c|}
\hline \multirow[t]{2}{*}{ Variables $^{a}$} & \multicolumn{2}{|c|}{ Hypocalcemia } & \multirow[t]{2}{*}{$p$ value } \\
\hline & Yes & No & \\
\hline Yes & $7(2.7)$ & $3(2.1)$ & \\
\hline \multicolumn{4}{|c|}{ Number of observed parathyroid glands, $n$ (\%) } \\
\hline 0 & $2(0.8)$ & 0 & \multirow[t]{5}{*}{$x^{2}=35.23, \mathrm{df}=4, p<0.001$} \\
\hline 1 & $3(1.2)$ & $5(3.5)$ & \\
\hline 2 & $76(29.6)^{* *}$ & $21(14.7)^{* *}$ & \\
\hline 3 & $142(55.3)$ & $66(46.2)$ & \\
\hline 4 & $34(13.2)^{* * *}$ & $51(36.7)^{* * *}$ & \\
\hline \multicolumn{4}{|c|}{ Upper branch of left inferior thyroid artery, $n(\%)$} \\
\hline Non-ligation & $231(89.9)$ & $132(92.3)$ & \multirow[t]{2}{*}{$x^{2}=0.64, \mathrm{df}=1, p=0.475$} \\
\hline Ligation & $26(10.1)$ & $11(7.7)$ & \\
\hline \multicolumn{4}{|c|}{ Lower branch of left inferior thyroid artery, $n(\%)$} \\
\hline Non-ligation & $232(90.3)$ & $134(93.7)$ & \multirow[t]{2}{*}{$x^{2}=1.39, \mathrm{df}=1, p=0.267$} \\
\hline Ligation & $25(9.7)$ & $9(6.3)$ & \\
\hline \multicolumn{4}{|c|}{ Trunk of left inferior thyroid artery, $n(\%)$} \\
\hline Non-ligation & $46(17.9)$ & $44(30.8)$ & \multirow[t]{2}{*}{$x^{2}=8.73, \mathrm{df}=1, p=0.003$} \\
\hline Ligation & $211(82.1)$ & $99(69.2)$ & \\
\hline \multicolumn{4}{|c|}{ Upper branch of right inferior thyroid artery, $n(\%)$} \\
\hline Non-ligation & $232(90.3)$ & $126(88.1)$ & \multirow[t]{2}{*}{$x^{2}=0.46, \mathrm{df}=1, p=0.501$} \\
\hline Ligation & $25(9.7)$ & $17(11.9)$ & \\
\hline \multicolumn{4}{|c|}{ Lower branch of right inferior thyroid artery, $n(\%)$} \\
\hline Non-ligation & $231(89.9)$ & $135(94.4)$ & \multirow[t]{2}{*}{$x^{2}=2.42, \mathrm{df}=1, p=0.137$} \\
\hline Ligation & $26(10.1)$ & $8(5.6)$ & \\
\hline \multicolumn{4}{|c|}{ Trunk of right inferior thyroid artery, $n(\%)$} \\
\hline Non-ligation & $44(17.1)$ & $47(32.9)$ & \multirow[t]{2}{*}{$x^{2}=12.96, \mathrm{df}=1, p<0.001$} \\
\hline Ligation & $213(82.9)$ & $96(67.1)$ & \\
\hline \multicolumn{4}{|c|}{ Number of darkened parathyroid glands at the end of surgery, $n(\%)$} \\
\hline 0 & $194(75.5)$ & $111(77.6)$ & \multirow[t]{4}{*}{$x^{2}=3.64, d f=3, p=0.303$} \\
\hline 1 & $54(21.0)$ & $26(18.2)$ & \\
\hline 2 & $7(2.7)$ & $2(1.4)$ & \\
\hline 3 & $2(0.8)$ & $4(2.8)$ & \\
\hline \multicolumn{4}{|c|}{ Parathyroid gland hematoma, $n$ (\%) } \\
\hline No & $244(94.9)$ & $135(94.4)$ & \multirow[t]{2}{*}{$x^{2}=0.05, \mathrm{df}=1, p=0.818$} \\
\hline Yes & $13(5.1)$ & $8(5.6)$ & \\
\hline \multicolumn{4}{|c|}{ Number of autotransplanted parathyroid glands, $n(\%)$} \\
\hline 0 & $205(79.8)^{*}$ & $127(88.8)^{*}$ & \multirow[t]{4}{*}{$x^{2}=6.32, \mathrm{df}=3, p=0.097$} \\
\hline 1 & $40(15.6)$ & $14(9.8)$ & \\
\hline 2 & $9(3.5)$ & $2(1.4)$ & \\
\hline 3 & $3(1.2)$ & 0 & \\
\hline \multicolumn{4}{|c|}{ Adenomatous nodules, $n$ (\%) } \\
\hline No & $218(84.8)$ & $116(81.1)$ & \multirow[t]{2}{*}{$x^{2}=0.92, \mathrm{df}=1, p=0.399$} \\
\hline Yes & $39(15.2)$ & $27(18.9)$ & \\
\hline Colloidal nodule & & & \\
\hline No & $81(31.5)$ & $49(34.3)$ & $x^{2}=0.32, \mathrm{df}=1, p=0.579$ \\
\hline
\end{tabular}


Table 2 Patient characteristics (Continued)

\begin{tabular}{|c|c|c|c|}
\hline \multirow[t]{2}{*}{ Variables $^{a}$} & \multicolumn{2}{|l|}{ Hypocalcemia } & \multirow[t]{2}{*}{$p$ value } \\
\hline & Yes & No & \\
\hline Yes & $176(68.5)$ & $94(65.7)$ & \\
\hline \multicolumn{4}{|l|}{ Papillary carcinoma, $n$ (\%) } \\
\hline No & $203(79.0)$ & $142(99.3)$ & \multirow[t]{2}{*}{$x^{2}=0.46, \mathrm{df}=1, p=0.518$} \\
\hline Yes & $54(21.0)$ & $26(18.2)$ & \\
\hline \multicolumn{4}{|l|}{ Follicular carcinoma, $n$ (\%) } \\
\hline No & $257(100)$ & $399(99.3)$ & \multirow[t]{2}{*}{$x^{2}=1.80, \mathrm{df}=1, p=0.358$} \\
\hline Yes & 0 & $1(0.7)$ & \\
\hline \multicolumn{4}{|l|}{ Medullary carcinoma, $n$ (\%) } \\
\hline No & $254(98.8)$ & $143(100)$ & \multirow[t]{2}{*}{$x^{2}=1.68, d f=1, p=0.556$} \\
\hline Yes & $3(1.2)$ & 0 & \\
\hline \multicolumn{4}{|l|}{ Anaplastic carcinoma, n (\%) } \\
\hline No & $257(100)$ & $143(100)$ & \\
\hline \multicolumn{4}{|l|}{ Autoimmune thyroiditis, $n$ (\%) } \\
\hline No & $234(91.1)$ & $129(90.2)$ & \multirow[t]{2}{*}{$X^{2}=0.08, \mathrm{df}=1, p=0.857$} \\
\hline Yes & $23(8.9)$ & $14(9.8)$ & \\
\hline Calcium day one, mmol/L, mean (SD) & $2.05(0.12)$ & $2.24(0.11)$ & \multirow[t]{2}{*}{$<0.001$} \\
\hline $\mathrm{MD}(\mathrm{IQR})$ & $2.05(0.16)$ & $2.25(0.13)$ & \\
\hline lonized calcium day one, $\mathrm{mmol} / \mathrm{L}$, mean (SD) & $0.96(0.07)$ & $1.06(0.07)$ & \multirow[t]{2}{*}{$<0.001$} \\
\hline $\mathrm{MD}(\mathrm{IQR})$ & $0.96(0.08)$ & $1.05(0.09)$ & \\
\hline PTH, ng/L (pg/mL), mean (SD) & $24.42(18.27)$ & $39.97(17.50)$ & \multirow[t]{2}{*}{$<0.001$} \\
\hline $\mathrm{MD}(\mathrm{IQR})$ & $21.47(29.24)$ & $38.09(20.86)$ & \\
\hline Calcium day two, mmol/L, mean (SD) & $1.96(0.12)$ & $2.20(0.08)$ & \multirow[t]{2}{*}{$<0.001$} \\
\hline $\mathrm{MD}(\mathrm{IQR})$ & $1.99(0.18)$ & $2.19(0.11)$ & \\
\hline lonized calcium day two, mmol/L, mean (SD) & $0.94(0.07)$ & $1.06(0.07)$ & \multirow[t]{2}{*}{$<0.001$} \\
\hline $\mathrm{MD}(\mathrm{IQR})$ & $0.94(0.09)$ & $1.05(0.08)$ & \\
\hline \multicolumn{4}{|l|}{ Revision for bleeding, $n$ (\%) } \\
\hline No & $254(98.8)$ & $142(99.3)$ & \multirow[t]{2}{*}{$x^{2}=0.20, \mathrm{df}=1, p=1.000$} \\
\hline Yes & $3(1.2)$ & $1(0.7)$ & \\
\hline \multicolumn{4}{|l|}{ Wound infection, $n(\%)$} \\
\hline No & $257(100)$ & $143(100)$ & \\
\hline \multicolumn{4}{|l|}{ Wound hematoma, $n$ (\%) } \\
\hline No & $249(96.9)$ & $140(97.9)$ & \multirow[t]{2}{*}{$x^{2}=0.35, \mathrm{df}=1, p=0.753$} \\
\hline Yes & $8(3.1)$ & $3(2.1)$ & \\
\hline \multicolumn{4}{|l|}{ Bleeding during surgery, $n$ (\%) } \\
\hline No & $256(99.6)$ & $143(100)$ & \multirow[t]{2}{*}{$x^{2}=0.56, \mathrm{df}=1, p=1.000$} \\
\hline Yes & $1(0.4)$ & 0 & \\
\hline \multicolumn{4}{|l|}{ Clinical voice hoarseness, $n$ (\%) } \\
\hline No & $245(95.3)$ & $139(97.2)$ & $x^{2}=0.84, \mathrm{df}=1, p=0.434$ \\
\hline Yes & $12(4.7)$ & $4(2.8)$ & \\
\hline Recurrent laryngeal nerve paralysis on one sid & & & \\
\hline No & $252(98.1)$ & $140(97.9)$ & $x^{2}=0.84, \mathrm{df}=1, p=0.434$ \\
\hline Yes & $5(1.9)$ & $3(2.1)$ & \\
\hline
\end{tabular}

Recurrent laryngeal nerve paralysis on both sides, ORL, $n$ (\%) 
Table 2 Patient characteristics (Continued)

\begin{tabular}{|c|c|c|c|}
\hline \multirow[t]{2}{*}{ Variables $^{a}$} & \multicolumn{2}{|c|}{ Hypocalcemia } & \multirow[t]{2}{*}{$p$ value } \\
\hline & Yes & No & \\
\hline No & $257(100)$ & $143(100)$ & \\
\hline \multicolumn{4}{|c|}{ Clinical manifestation of hypocalcemia, $n(\%)$} \\
\hline None & $197(76.7)$ & $143(100)$ & \multirow[t]{3}{*}{$x^{2}=39.27, \mathrm{df}=2, p<0.001$} \\
\hline Day 1 & $14(3.5)$ & 0 & \\
\hline Day 2 & $46(17.9)$ & 0 & \\
\hline \multicolumn{4}{|c|}{ Hypocalcemia without clinical manifestation, $n(\%)$} \\
\hline No & $60(23.3)$ & $143(100)$ & \multirow[t]{2}{*}{$x^{2}=215.99, \mathrm{df}=1, p<0.001$} \\
\hline Yes & $197(76.7)$ & 0 & \\
\hline \multicolumn{4}{|c|}{ Hypocalcemia - Chvostek's symptoms, n (\%) } \\
\hline No & $243(94.6)$ & $143(100)$ & \multirow[t]{2}{*}{$x^{2}=8.07, \mathrm{df}=1, p=0.004$} \\
\hline Yes & $14(5.4)$ & 0 & \\
\hline \multicolumn{4}{|c|}{ Hypocalcemia - Trousseau's symptoms, n (\%) } \\
\hline No & $249(96.9)$ & $143(100)$ & \multirow[t]{2}{*}{$x^{2}=4.54, \mathrm{df}=1, p=0.033$} \\
\hline Yes & $8(2.0)$ & 0 & \\
\hline \multicolumn{4}{|c|}{ Hypocalcemia - Numbness of fingers, $n(\%)$} \\
\hline No & $199(77.4)$ & $143(100)$ & \multirow[t]{2}{*}{$x^{2}=37.75, \mathrm{df}=1, p<0.001$} \\
\hline Yes & $58(22.6)$ & 0 & \\
\hline \multicolumn{4}{|c|}{ Treatment commenced in hospital with calcium and vitamin D preparations after surgery, $n(\%)$} \\
\hline None & $23(8.9)$ & $139(97.2)$ & \multirow[t]{5}{*}{$x^{2}=297.04, \mathrm{df}=4, p<0.001$} \\
\hline Day 1 & $15(5.8)$ & 0) & \\
\hline Day 2 & $33(12.8)$ & 0 & \\
\hline Day 3 & $5(1.9)$ & 0 & \\
\hline On discharge & $181(70.3)$ & $4(2.8)$ & \\
\hline
\end{tabular}

${ }^{a}$ Continuous variables are presented as means and standard deviations (SD) \& medians and interquartile ranges (IQR); categorical variables are presented as $n(\%)$. ${ }^{\mathrm{b}}$ Student $\mathrm{t}$ test; ${ }^{\mathrm{C}}$ Mann-Whitney $\mathrm{U}$ test. ${ }^{*} p<0.05$, $\mathrm{z}$ test, ${ }^{* *} p<0.01,{ }^{* * *} p<0.001$. PTH Parathyroid hormone, ORL otorhinolaryngologist

of the development of hypocalcemia. The greater the number of PGs found during surgery, the lower the chance of hypocalcemia. The study by Thomusch et al. determined that permanent post-operative hypocalcemia was more likely to develop if less than two PGs were found during surgery [40]. On the other hand, other studies have found that more PGs found during surgery may be associated with temporary hypocalcemia $[12,25,41]$.

In our study, the number of PGs autotransplanted during surgery was a statistically reliable predictor of the development of post-TT hypocalcemia $(p=0.015)$. The greater the number of autotransplanted PGs, the higher the chance of hypocalcemia. Several studies have shown that autotransplantation of one or more PGs results in a far higher chance of hypocalcemia [12, 17, 18, 42]. However, several other studies showed no association between autotransplantation of PGs and permanent hypocalcemia [18, 25, 31, 43].

In our study, thyrotoxicosis $<10$ years before surgery was found to be a statistically reliable predictor of post-TT hypocalcemia $(p=0.046)$. Thyrotoxicosis $>10$ years before surgery was not significantly associated with post-TT hypocalcemia $(p=0.982)$; however, there were only 18 such patients $(4.5 \%)$ in our study. Based on our data, Graves' disease was not a statistically significant predictor of post-TT hypocalcemia $(p=0.247)$, but again, it was only diagnosed in 21 patients (5.3\%). The multivariate analysis by Thomusch et al. demonstrated that, in patients with Graves' disease, both temporary and permanent hypocalcemia developed more often following TT [40]. Further, a meta-analysis of four studies with 6681 patients showed a greater frequency of post-operative temporary hypocalcemia in patients with Graves' disease [40, 44-46].

Ligation of the trunk of the left inferior thyroid artery $(p=0.003)$ and the trunk of the right inferior thyroid artery $(p<0.001)$ were found to be statistically reliable predictors of hypocalcemia in our study. In a study of 1411 patients, Park et al. found that saving vessels supplying blood to the thyroid gland during surgery reduced the frequency of both temporary and permanent hypocalcemia in univariate $(p=0.01)$ and multivariate 
Table 3 Associations between patient characteristics and risk of hypocalcemia - Univariate model

\begin{tabular}{|c|c|c|c|}
\hline Variable & OR & $95 \% \mathrm{Cl}$ & $p$ \\
\hline Thyrotoxicosis before surgery: no & 1 & & 0.132 \\
\hline$<10$ years & 1.65 & $1.01-2.70$ & 0.046 \\
\hline$>10$ years & 0.99 & $0.37-2.63$ & 0.982 \\
\hline Thyroidectomy without lymphadenectomy & 0.63 & $0.35-1.13$ & 0.118 \\
\hline Thyroidectomy with one sided central lymphadenectomy & 1.62 & $0.86-3.04$ & 0.134 \\
\hline Thyroidectomy with central lymphadenectomy on both sides & 1.31 & $0.33-5.13$ & 0.702 \\
\hline Upper branch of left inferior thyroid artery, ligation & 0.74 & $0.35-1.55$ & 0.424 \\
\hline Lower branch of left inferior thyroid artery, ligation & 0.62 & $0.28-1.38$ & 0.241 \\
\hline Trunk of left inferior thyroid artery, ligation & 2.04 & $1.27-3.29$ & 0.003 \\
\hline Upper branch of right inferior thyroid artery, ligation & 1.25 & $0.65-2.41$ & 0.500 \\
\hline Lower branch of right inferior thyroid artery, ligation & 1.90 & $0.84-4.31$ & 0.125 \\
\hline Trunk of right inferior thyroid artery, ligation & 2.37 & $1.47-3.82$ & $<0.001$ \\
\hline Number of darkened parathyroid glands at the end of surgery & 0.98 & $0.69-1.39$ & 0.925 \\
\hline Parathyroid gland hematoma & 0.90 & $0.36-2.22$ & 0.818 \\
\hline Adenomatous nodules & 0.77 & $0.45-1.32$ & 0.339 \\
\hline Colloidal nodules & 1.13 & $0.73-1.75$ & 0.574 \\
\hline Papillary carcinoma & 1.20 & $0.71-2.01$ & 0.498 \\
\hline Autoimmune thyroiditis & 0.91 & $0.45-1.82$ & 0.781 \\
\hline Graves' disease & 0.55 & $0.20-1.52$ & 0.247 \\
\hline Calcium before surgery & 0.01 & $1.01-0.57$ & $<0.001$ \\
\hline Calcium, day 1 after surgery & 0.00 & $0.00-0.00$ & $<0.001$ \\
\hline Calcium, day 2 after surgery & 0.00 & $0.00-0.00$ & $<0.001$ \\
\hline lonized calcium before surgery & 0.001 & $0.000-0.013$ & $<0.001$ \\
\hline Ionized calcium, day 1 after surgery & 0.00 & $0.00-0.00$ & $<0.001$ \\
\hline Ionized calcium, day 2 after surgery & 0.00 & $0.00-0.00$ & $<0.001$ \\
\hline PTH before surgery & 1.00 & $0.99-1.01$ & 0.854 \\
\hline PTH, day 1 after surgery & 0.96 & $0.94-0.97$ & $<0.001$ \\
\hline 25-hydroxyvitamin D before surgery & 0.99 & $0.99-1.00$ & 0.201 \\
\hline TSH before surgery & 1.13 & $0.94-1.36$ & 0.197 \\
\hline FT4 before surgery & 0.98 & $0.91-1.06$ & 0.625 \\
\hline FT3 before surgery & 0.99 & $0.91-1.08$ & 0.854 \\
\hline
\end{tabular}

PTH Parathyroid hormone, TSH thyroid stimulating hormone, FT3 free triiodothyronine, FT4 free thyroxine

$(p=0.02)$ analyses [47]. According to Lee et al., saving veins on both sides of the thyroid may reduce post-TT hypocalcemia [48]. It has long been accepted that post-TT hypocalcemia depends on the experience of the surgeon and the surgical technique

Table 4 Associations between patient characteristics and risk of hypocalcemia - Multivariate model

\begin{tabular}{llll}
\hline Variable & OR & $95 \% \mathrm{Cl}$ & $p$ \\
\hline Age & 1.05 & $1.01-1.09$ & 0.029 \\
Gender & 5.94 & $1.13-31.26$ & 0.035 \\
Calcium, second day after surgery & 0.00 & $0.00-0.00$ & $<0.001$ \\
\hline
\end{tabular}

OR Odds ratio, $\mathrm{Cl}$ confidence interval used $[49,50]$. Our study identified some risk factors related to the surgical technique; hence, it is important not only to identify important factors following surgery that may influence development of hypocalcemia, but also to reconsider the surgical technique in order to minimize this risk, if possible.

In our study, the frequency analysis showed that neither gender $(p=0.351)$ nor age $(p=0.207)$ had a significant impact on development of hypocalcemia. However, in the multivariate analysis, female gender $(p$ $=0.035)$ and older age $(p=0.029)$ were statistically significant predictors. There is no definitive evidence on the impact of age on the development of post-operative hypocalcemia in the literature. Some studies suggest that 
temporary post-operative hypocalcemia is more common in younger patients $[15,25]$, while others suggest it is more common in older patients [19, 21]. A meta-analysis of five studies with 2576 patients revealed no significant association between patient age and temporary hypocalcemia $[15,17-19,21]$. While a meta-analysis of 10 studies involving 3443 patients showed that temporary postoperative hypocalcemia was more common in women [12, $15,17-21,23,41,51]$.

\section{Conclusions}

This study demonstrates that there is a number of different patient (gender, age, and thyrotoxicosis $<10$ years before surgery) and surgical (number of PGs found observed surgery, decreased $\mathrm{Ca}$ and iCa before surgery, PTH on day one following surgery, and ligation of the trunk of the left and right inferior thyroid artery) risk factors predictive of the development of hypocalcemia following TT. Our study suggests that it is important not only to identify factors following surgery that may predict development of hypocalcemia, but also to reconsider the surgical technique, in order to minimize this risk, if possible. Identification of known risk factors post-operatively could allow for early detection and effective treatment of these patients.

\section{Abbreviations}

25-hydroxy Vit D: 25-hydroxyvitamin D; Ca: Calcium; Cls: Confidence Intervals; iCa: ionized Calcium; LR: Likelihood Ratio; ORs: Odds Ratios; PGs: Parathyroid Glands; PTH: Parathyroid Hormone; TT: Total Thyroidectomy

\section{Availability of data and materials}

The datasets generated and analysed during the current study are available from the corresponding author on reasonable request.

\section{Authors' contributions}

Conceived of the study, designed study, collected data, wrote article, revised article, interpretated of data, participated in coordination: VE. Designed study, collected data, revised article, interpretated of data, participated in coordination: VB. Designed study, collected data, revised article, interpretated of data, participated in coordination: AS. Made substantial contributions to conception and design, interpretated of data, revised the manuscript critically for important intellectual content: VJ, KS, ZD, AG, PZ. Made substantial contributions to conception and design, analyzed data, interpretated of data: AM. All authors read and approved the final manuscript.

\section{Ethics approval and consent to participate}

This study was approved by Lithuanian Bioethics Committee (2014-12-02 No.: L-14-09/1, No.: L-14-09/2). Informed consent was obtained from all individual participants included in the study. The informed consent was in written.

\section{Consent for publication}

Consent for publication was obtained from all individual participants included in the study. Consent for publication was in written.

\section{Competing interests}

The authors declare that they have no competing interests.

\section{Publisher's Note}

Springer Nature remains neutral with regard to jurisdictional claims in published maps and institutional affiliations.

\section{Author details}

'Department of Abdominal and Endocrine Surgery, Klaipeda University Hospital, Liepojos St. 41, 92288 Klaipeda, Lithuania. ${ }^{2}$ Centre of Abdominal Surgery, Vilnius University Hospital Santaros Klinikos, Vilnius, Lithuania. ${ }^{3}$ Department of Surgery, Hospital of Lithuanian University of Health Sciences, Kaunas, Lithuania. ${ }^{4}$ Department of Medical Technologies, Faculty of Health Sciences, Klaipeda University, Klaipeda, Lithuania.

Received: 15 April 2018 Accepted: 30 July 2018

Published online: 09 August 2018

\section{References}

1. Karamanakos SN, Markuo KB, Panagoppulos K, Karavias D, Vagianos CE, Scopa CD, et al. Complications and risk factors related to the extent of surgery in thyroidectomy-results from 2,043 procedures. Hormones. 2010;9: $318-25$.

2. Ho TW, Shaheen AA, Dixon E, Harvey A. Utilization of thyroidectomy for benign disease in the United States: a 15-year population-based study. Am J Surg. 2011;201:570-4.

3. Rosato L, Avenia N, Bernante P, De Palma M, Gulino G, Nasi PG, et al. Complications of thyroid surgery: analysis of a multicentric study on 14,934 patients operated on in Italy over 5 years. World J Surg. 2004;28:271-6.

4. Wilson RB, Erskine C, Crowe PJ. Hypomagnesemia and hypocalcemia after thyroidectomy: a prospective study. World J Surg. 2000;24:722-6.

5. Jacobs JK, Aland JW Jr, Ballinger JF. Total thyroidectomy. A review of 213 patients. Ann Surg. 1983;197:542-9.

6. Reeve T, Thompson NW. Complications of thyroid surgery: how to avoid them, how to manage them, and observations on their possible effect on the whole patient. World J Surg. 2000;24:971-5.

7. D'Ajello F, Cirocchi R, Docimo G, Catania A, Ardito G, Rosato L, et al. Thyroidectomy with ultrasonic dissector: a multicentric experience. G Chir. 2010;31(6-7):289-92.

8. Stack BC, Bimston DN, Bodenner DL, Brett EM, Dralle H, Orloff LA, et al. AACE/ACE disease state clinical review: postoperative hypoparathyroidism definitions and management. Endocr Pract. 2015;21(6):674-85.

9. Edafe O, Antakis R, Laskar N, Uttley L, Balasubramanian SP. Systematic review and meta-analysis of predictors of post-thyroidectomy hypocalcaemia. Br J Surg. 2014;101(4):307-20.

10. Demeester-Mirkine N, Hooghe L, Van Geertruyden J, De Maetelaer V. Hypocalcemia after thyroidectomy. Arch Surg. 1992;127:854-8.

11. Lemaire FX, Debruyne F, Delaere P, Vander Porten V. Parathyroid function in the early postoperative period after thyroidectomy. Acta Otorhinolaryngol Belg. 2001;55:187-97.

12. Lang BH, Yih PC, Ng KK. A prospective evaluation of quick intraoperative parathyroid hormone assay at the time of skin closure in predicting clinically relevant hypocalcemia after thyroidectomy. World I Surg. 2012;36: 1300-6.

13. Ali S, Yu C, Palmer FL, Ganly I, Shaha A, Shah JP, et al. Nomogram to aid selection of patients for short-stay thyroidectomy based on risk of postoperative hypocalcemia. Arch Otolaryngol Head Neck Surg. 2011;137: 1154-60.

14. Amir A, Sands NB, Tamilia M, Hier MP, Black MJ, Payne RJ. Preoperative serum calcium levels as an indicator of postthyroidectomy hypocalcemia. J Otolaryngol Head Neck Surg. 2010;39:654-8.

15. Yamashita H, Noguchi S, Tahara K, Watanabe S, Uchino S, Kawamoto H, et al. Postoperative tetany in patients with graves' disease: a risk factor analysis. Clin Endocrinol. 1997;47:71-7.

16. Gentileschi P, Gacek IA, Manzelli A, Cascarella G, Sileri P, Lirosi F, et al. Early (1 hour) post-operative parathyroid hormone (PTH) measurement predicts hypocalcaemia after thyroidectomy: a prospective case-control singleinstitution study. Chir Ital. 2008;60:519-28.

17. Moriyama T, Yamashita H, Noguchi S, Takamatsu Y, Ogawa T, Watanabe S, et al. Intraoperative parathyroid hormone assay in patients with graves' disease for prediction of postoperative tetany. World I Surg. 2005;29:1282-7.

18. Sitges-Serra A, Ruiz S, Girvent M, Manjón H, Dueñas JP, Sancho JJ. Outcome of protracted hypoparathyroidism after total thyroidectomy. Br J Surg. 2010; 97:1687-95.

19. Kamer E, Unalp HR, Erbil Y, Akguner T, Issever H, Tarcan E. Early prediction of hypocalcemia after thyroidectomy by parathormone measurement in surgical site irrigation fluid. Int J Surg. 2009;7:466-71. 
20. Erbil Y, Barbaros U, Temel B, Turkoglu U, Issever $\mathrm{H}$, Bozbora A, et al. The impact of age, vitamin $\mathrm{D}(3)$ level, and incidental parathyroidectomy on. Am J Surg. 2009;197:439-46.

21. Erbil Y, Bozbora A, Ozbey N, Issever H, Aral F, Ozarmagan S, et al. Predictive value of age and serum parathormone and vitamin D3 levels for postoperative hypocalcemia after total thyroidectomy for nontoxic multinodular goiter. Arch Surg. 2007;142:1182-7.

22. Walsh SR, Kumar B, Coveney EC. Serum calcium slope predicts hypocalcaemia following thyroid surgery. Int J Surg. 2007:5:41-4.

23. Yamashita H, Murakami T, Noguchi S, Shiiba M, Watanabe S, Uchino S, et al, Postoperative tetany in graves disease: important role of vitamin $D$ metabolites. Ann Surg. 1999;229:237-45.

24. Luu Q, Andersen PE, Adams J, Wax MK, Cohen J. The predictive value of perioperative calcium levels after thyroid/parathyroid surgery. Head Neck. 2002;24:63-7.

25. Hallgrimsson $P$, Nordenström $E$, Almquist $M$, Bergenfelz AO. Risk factors for medically treated hypocalcemia after surgery for graves' disease: a Swedish multicenter study of 1,157 patients. World J Surg. 2012;36:1933-42.

26. Díez Alonso M, Sánchez López JD, Sánchez-Seco Peña MI, Ratia Jimenez T, Arribas Gomez I, Rodriguez Pascual A, et al. Serum PTH levels as a predictive factor of hypocalcaemia after total thyroidectomy. Cir Esp. 2009;85:96-102.

27. Graff AT, Miller FR, Roehm CE, Prihoda TJ. Predicting hypocalcemia after total thyroidectomy: parathyroid hormone level vs. serial calcium levels. Ear Nose Throat J. 2010;89:462-5.

28. Alia P, Moreno P, Rigo R, Francos JM, Navarro MA. Postresection parathyroid hormone and parathyroid hormone decline accurately predict hypocalcemia after thyroidectomy. Am J Clin Pathol. 2007;127:592-7.

29. Wong C, Price S, Scott-Coombes D. Hypocalcaemia and parathyroid hormone assay following total thyroidectomy: predicting the future. World $J$ Surg. 2006;30:825-32.

30. Roh JL, Park Cl. Intraoperative parathyroid hormone assay for management of patients undergoing total thyroidectomy. Head Neck. 2006;28:990-7.

31. Aluffi P, Aina E, Bagnati T, Toso A, Pia F. Prognostic factors for definitive hypoparathyroidism following total thyroidectomy. Acta Otorrinolaringol Esp. 2008;59:321-4.

32. Pisanu A, Cois A, Piu S, Altana ML, Uccheddu A. Factors predicting outcome of hypocalcaemia following total thyroidectomy. Chir Ital. 2003:55:35-40.

33. Pattou F, Combemale F, Fabre S, Carnaille B, Decoulx M, Wemeau JL, et al. Hypocalcemia following thyroid surgery: incidence and prediction of outcome. World J Surg. 1998;22:718-24.

34. Tartaglia F, Giuliani A, Sgueglia M, Patrizi G, Di Rocco G, Blasi S, et al. Is ionized calcium a reliable predictor of hypocalcemia after total thyroidectomy? A before and after study. G Chir. 2014;35(1/2):27-35.

35. Chapman DB, French CC, Leng X, Browne JD, Waltonen JD, Sullivan CA. Parathyroid hormone early percent change: an individualized approach to predict postthyroidectomy hypocalcemia. Am J Otolaryngol. 2012;33:216-20.

36. Kovacevic B, Ignjatovic M, Cuk V, Zivaljevic V, Paunovic I. Early prediction of symptomatic hypocalcemia after total thyroidectomy. Acta Chir Belg. 2011;111:303-7.

37. Proczko-Markuszewska M, Kobiela J, Stefaniak T, Lachiński AJ, Sledziński Z. Postoperative PTH measurement as a predictor of hypocalcaemia after thyroidectomy. Acta Chir Belg. 2010;110:40-4.

38. Vanderlei FA, Vieira JG, Hojaij FC, Cervantes O, Kunii IS, Ohe MN, et al. Parathyroid hormone: an early predictor of symptomatic hypocalcemia after total thyroidectomy. Arq Bras Endocrinol Metabol. 2012:56:168-72.

39. Lecerf P, Orry D, Perrodeau E, Lhommet C, Charretier C, Mor C, et al Parathyroid hormone decline 4 hours after total thyroidectomy accurately predicts hypocalcemia. Surgery. 2012;152:863-8.

40. Thomusch O, Machens A, Sekulla C, Ukkat J, Brauckhoff M, Dralle $\mathrm{H}$. The impact of surgical technique on postoperative hypoparathyroidism in bilateral thyroid surgery: a multivariate analysis of 5846 consecutive patients. Surgery. 2003;133:180-5

41. Pfleiderer AG, Ahmad N, Draper MR, Vrotsou K, Smith WK. The timing of calcium measurements in helping to predict temporary and permanent hypocalcaemia in patients having completion and total thyroidectomies. Ann R Coll Surg Engl. 2009:91:140-6.

42. Asari R, Passler C, Kaczirek K, Scheuba C, Niederle B. Hypoparathyroidism after total thyroidectomy: a prospective study. Arch Surg. 2008;143:132-7.

43. Promberger $\mathrm{R}, \mathrm{Ott} J$, Kober $\mathrm{F}$, Mikola B, Karik M, Freissmuth $\mathrm{M}$, et al. Intraand postoperative parathyroid hormone-kinetics do not advocate for autotransplantation of discolored parathyroid glands during thyroidectomy. Thyroid. 2010;20:1371-5.

44. Serpell JW, Phan D. Safety of total thyroidectomy. ANZ J Surg. 2007;77:15-9.

45. Welch KC, McHenry CR. Total thyroidectomy: is morbidity higher for graves' disease than nontoxic goiter? J Surg Res. 2011;170:96-9.

46. Chiang FY, Lin JC, Wu CW, Lee KW, Lu SP, Kuo WR, et al. Morbidity after total thyroidectomy for benign thyroid disease: comparison of graves' disease and non-graves' disease. Kaohsiung J Med Sci. 2006;22:554-9.

47. Park I, Rhu J, Woo J-W, Choi JH, Kim JS, Kim JH. Preserving parathyroid gland vasculature to reduce post-thyroidectomy hypocalcemia. World J Surg. 2016:40:1382-9.

48. Lee DY, Cha W, Jeong WJ, Ahn SH. Preservation of the inferior thyroidal vein reduces post-thyroidectomy hypocalcemia. Laryngoscope. 2014;124:1272-7.

49. Sosa JA, Bowman HM, Tielsch JM, Powe NR, Gordon TA, Udelsman R. The importance of surgeon experience for clinical and economic outcomes from thyroidectomy. Ann Surg. 1998;228(3):320-30.

50. Kandil E, Noureldine SI, Abbas A, Tufano RP. The impact of surgical volume on patient outcomes following thyroid surgery. Surgery. 2013;154(6):1346-53.

51. Sands NB, Payne RJ, Cote V, Hier MP, Black MJ, Tamilia M. Female gender as a risk factor for transient post-thyroidectomy hypocalcemia. Otolaryngol Head Neck Surg. 2011;145:561-4

\section{Ready to submit your research? Choose BMC and benefit from:}

- fast, convenient online submission

- thorough peer review by experienced researchers in your field

- rapid publication on acceptance

- support for research data, including large and complex data types

- gold Open Access which fosters wider collaboration and increased citations

- maximum visibility for your research: over $100 \mathrm{M}$ website views per year

At BMC, research is always in progress.

Learn more biomedcentral.com/submissions 